\title{
Total and Viscosity Cross Sections for Krypton Gas at Boiling Point
}

\author{
Ibtisam. F. Al-Maaitah ${ }^{1}$ \\ ${ }^{1}$ Applied Physics Department, Tafila Technical University, Tafila, Jordan \\ Correspondence: Ibtisam. F. Al-Maaitah, Applied Physics Department, Tafila Technical University, Tafila, \\ Jordan. E-mail: ibt.f@yahoo.com \\ Received: February 18, 2019 \\ Accepted: March 14, 2019 \\ Online Published: March 30, 2019 \\ doi:10.5539/apr.v11n2p88 \\ URL: http://dx.doi.org/10.5539/apr.v11n2p88
}

\begin{abstract}
We have calculated total and viscosity cross sections for krypton gas at boiling point by using a Galitskii-Migdal-Feynman (GMF) formalism which is essentially an independent-pair model 'dressed' by a many-body medium. The interaction potential in our work is theHartree-Fock dispersion (HFD-B) potential.
\end{abstract}

Keywords: Galitskii-Migdal-Feynman, Scattering, Viscosity Cross Section

\section{Introduction}

Viscosity cross sections for argon, krypton and xenon from zero to $1 \mathrm{keV}$ using the phase shifts was calculated (Robert \& Allan, 2015). In this work, we shall invoke the Galitskii-Migdal-Feynman (GMF) T-matrix to calculate the effective phase shifts in the medium and then use them to calculate the effective viscosity cross section at different temperature. The properties of the interatomic krypton potential are:(i) The repulsive term, describes Pauli repulsion at short ranges due to overlapping electron orbitals; (ii) The attractive long-range term, describes attraction at long ranges; (iii) The interaction energy is a minimum at the equilibrium position. The results are presented and discussed.

\section{Theoretical Framework}

Total and viscosity Cross Sections: We start with boson-boson scattering in a medium. A spinless boson with wave vector $\overrightarrow{\mathrm{k}}$ and orbital angular momentum $\vec{\ell}$ is incident on another spinless boson initially at rest in the medium. General expressions for the cross sections, including the total $\left(\sigma_{\mathrm{T}}\right)$ and viscosity $\left(\sigma_{\eta}\right)$ cross sections, are given by (Geltman, 1997; Merzbacher, 1998; Kanzleiter et al., 2000; Wright et al., 2005; Joudeh, 2013)

$$
\begin{gathered}
\sigma_{\mathrm{T}}=\frac{8 \pi}{\mathrm{k}^{2}} \sum_{\ell(\text { even })}^{\infty}(2 \ell+1) \sin ^{2}\left(\delta_{\ell}^{\mathrm{E}}(\mathrm{k})\right) \\
\sigma_{\mathrm{\eta}}=\frac{4 \pi}{\mathrm{k}^{2}} \sum_{\ell \text { (even })}^{\infty} \frac{(\ell+1)(\ell+2)}{\left(\ell+\frac{3}{2}\right)} \sin ^{2}\left(\delta_{\ell+2}^{\mathrm{E}}(\mathrm{k})-\delta_{\ell}^{\mathrm{E}}(\mathrm{k})\right)
\end{gathered}
$$

The starting point in computing $\sigma_{\mathrm{T}}$ and $\sigma_{\eta}$ is the determination of $\delta_{\ell}^{\mathrm{E}}$. This can be accomplished by solving the GMF integral equation for the T-matrix, using a matrix-inversion technique. This matrix is essentially an effective pairwise interaction in momentum space. It can also be viewed as a generalized scattering amplitude, or as a 'dressed' Lippmann-Schwinger (LS) t-matrix (which describes the scattering of two particles in free space). The GMF T-matrix was originally derived for many-fermionic systems; but it was later adapted to many-bosonic systems (Al-Barghouthi, 1997).

This matrix is given by a Bethe-Salpeter-like equation (Fetter \& Walecka, 1971; Ghassib et al., 1976; Bishop et al., 1976): 


$$
\left.\left.\mathrm{T}\left(\overrightarrow{\mathrm{p}}, \overrightarrow{\mathrm{p}}^{\prime} ; \mathrm{s}, \overrightarrow{\mathrm{P}}, \beta\right)=\mathrm{u}(\overrightarrow{\mathrm{p}}-\overrightarrow{\mathrm{p}} \mid)-(2 \pi)^{-3} \int \mathrm{d} \overrightarrow{\mathrm{k} u}(\overrightarrow{\mathrm{p}}-\overrightarrow{\mathrm{k}})\right) \times \mathrm{g}_{0}(\mathrm{k}, \mathrm{s}) \mathrm{Q}(\overrightarrow{\mathrm{k}}, \overrightarrow{\mathrm{P}}, \beta)-\mathrm{g}_{0}^{+}(\mathrm{k}, \mathrm{s}) \overline{\mathrm{Q}}(\overrightarrow{\mathrm{k}}, \overrightarrow{\mathrm{P}}, \beta)\right] \mathrm{T}\left(\overrightarrow{\mathrm{k}}, \overrightarrow{\mathrm{p}}^{\prime} ; \mathrm{s}, \overrightarrow{\mathrm{P}}, \beta\right)
$$

Here: $\mathrm{p}$ and $\mathrm{p}^{\prime}$ are the relative incoming and outgoing momenta; $\mathrm{P}$ is the center-of-mass momentum. The operator $\mathrm{u} \equiv \frac{2 \mathrm{~m}_{\mathrm{r}} \mathrm{V}}{\hbar^{2}} \equiv \frac{1}{2} \mathrm{~V}$ [in natural units], and $\mathrm{V}$ the Fourier transform of a static central two-body potential. The free two-body Green's function go(s) is specified by $\mathrm{g}_{0}(\overrightarrow{\mathrm{k}}, \mathrm{s}) \equiv \frac{1}{\mathrm{k}^{2}-\mathrm{s}-\mathrm{i \eta}}$, $\eta$ being a positive infinitesimal in the scattering region $(\mathrm{s}>0)$ and zero otherwise, and the parameter $\mathrm{S}$ the total energy of the interacting pair in the center-of-mass frame, given by $\mathrm{s} \equiv 2 \mu\left(2 \mathrm{P}_{\mathrm{o}}-\frac{\mathrm{P}^{2}}{\mathrm{~m}}\right)$

$\mathrm{P}_{0}$ is the total energy of the pair and $\mathrm{P}^{2}$ is the energy carried by the center of mass.

where $n(\vec{k})$ is the Bose-Einstein distribution: $n(\varepsilon)=\frac{1}{\mathrm{e}^{\beta(\varepsilon-\mu)}-1}, \varepsilon$ being the single-particle energy.

The chemical potential $\mu$ is given by (Kittel and Kroemer, 1980) $\mu=k_{B} T \ln \left(\frac{n}{n_{q}}\right), n_{q}$ being the quantum concentration, given by $\mathrm{n}_{\mathrm{q}}=\left(\frac{\mathrm{mk}_{\mathrm{B}} \mathrm{T}}{2 \pi \hbar^{2}}\right)^{3 / 2}$.

Upon partial-wave decomposition, Equation (3) takes the form (Bishop et al., 1976)

$$
\left.\mathrm{T}_{\ell}\left(\overrightarrow{\mathrm{p}}, \overrightarrow{\mathrm{p}}^{\prime} ; \mathrm{s}, \overrightarrow{\mathrm{P}}, \beta\right)=\mathrm{u}_{\ell}(|\overrightarrow{\mathrm{p}}-\overrightarrow{\mathrm{p}}|)-(2 \pi)^{-2} \int_{0}^{\infty} \mathrm{k}^{2} \mathrm{~d} \overrightarrow{\mathrm{k}} \mathrm{u}_{\ell}(|\overrightarrow{\mathrm{p}}-\overrightarrow{\mathrm{k}}|) \times \mathrm{g}_{0}(\mathrm{k}, \mathrm{s}) \mathrm{Q}(\overrightarrow{\mathrm{k}}, \overrightarrow{\mathrm{P}}, \beta)-\mathrm{g}_{0}^{+}(\mathrm{k}, \mathrm{s}) \overline{\mathrm{Q}}(\overrightarrow{\mathrm{k}}, \overrightarrow{\mathrm{P}}, \beta)\right] \mathrm{T}_{\ell}\left(\overrightarrow{\mathrm{k}}, \overrightarrow{\mathrm{p}}^{\prime} ; \mathrm{s}, \overrightarrow{\mathrm{P}}, \beta\right)(4)
$$

This equation represents the full off-shell T-matrix pertaining to a relative partial wave $\ell$, from which the on-energy-shell counterpart $T_{\ell}(\overrightarrow{\mathrm{p}}, \overrightarrow{\mathrm{P}})$ is obtained directly by setting $\overrightarrow{\mathrm{p}}=\overrightarrow{\mathrm{p}}^{\prime}$ and $\mathrm{s}=\mathrm{p}^{2}$. Clearly, in the free-scattering limit, $\mathrm{Q}(\overline{\mathrm{Q}}) \rightarrow 1(0)$; so that Equation (3) reduces to the LS T-matrix.

The parameterized $T_{\ell}(p ; P ; \beta)$ can be expressed in terms of real many-body shifts $\delta_{\ell}^{E}(p ; P, \beta)$ according to (Ghassib et al., 1976; Bishop et al., 1976)

$$
\mathrm{T}_{\ell}(\mathrm{p} ; \mathrm{P} ; \beta)=-\frac{2 \pi}{\mathrm{p}(\mathrm{Q}(\mathrm{p} ; \mathrm{P}, \beta)+\overline{\mathrm{Q}}(\mathrm{p} ; \mathrm{P}, \beta))}\left[\sin \left(2 \delta_{\ell}^{\mathrm{E}}(\mathrm{p} ; \mathrm{P}, \beta)\right)+\mathrm{i}\left(1-\cos \left(2 \delta_{\ell}^{\mathrm{E}}(\mathrm{p} ; \mathrm{P}, \beta)\right)\right)\right]
$$

so that

$$
\delta_{\ell}^{\mathrm{E}}(\mathrm{p} ; \mathrm{P}, \beta) \equiv \tan ^{-1} \frac{\operatorname{ImT}_{\ell}(\mathrm{p} ; \mathrm{P} ; \beta)}{\operatorname{ReT}_{\ell}(\mathrm{p} ; \mathrm{P} ; \beta)}
$$

$\operatorname{ImT}_{\ell}(p ; P ; \beta)$ and $\operatorname{ReT}_{\ell}(p ; P ; \beta)$ denote, respectively, the imaginary and real parts of $T_{\ell}(p ; P ; \beta)$; they are defined by

$$
\operatorname{Re}_{\ell}(\mathrm{p} ; \mathrm{P} ; \beta)=-\frac{2 \pi}{\mathrm{p}(\mathrm{Q}(\mathrm{p} ; \mathrm{P}, \beta)+\overline{\mathrm{Q}}(\mathrm{p} ; \mathrm{P}, \beta))} \sin \left(2 \delta_{\ell}^{E}(\mathrm{p} ; \mathrm{P}, \beta)\right)
$$




$$
\operatorname{ImT}(\mathrm{p} ; \mathrm{P} ; \beta)=-\frac{2 \pi}{\mathrm{p}(\mathrm{Q}(\mathrm{p} ; \mathrm{P}, \beta)+\overline{\mathrm{Q}}(\mathrm{p} ; \mathrm{P}, \beta))}\left(1-\cos \left(2 \delta_{\ell}^{\mathrm{E}}(\mathrm{p} ; \mathrm{P}, \beta)\right)\right)
$$

The two-body potential representing the $\mathrm{Kr}-\mathrm{Kr}$ interaction is taken in the present work as the HFD-B potential (Ronald \& Slaman, 1986).

given by $\mathrm{V}(\mathrm{r})=\varepsilon \mathrm{V}^{*}(\mathrm{x})$, where

$$
\begin{aligned}
& V^{*}(x)=A^{*} \exp \left(-\alpha^{*} x+\beta^{*} x^{2}\right)-F(x) \sum_{j=0}^{2} c_{2 j+6} / x^{2 j+6} \\
& \mathrm{~F}(\mathrm{x})= \begin{cases}\exp -\left[\left\{\frac{\mathrm{D}}{\mathrm{x}}-1\right\}^{2}\right], & \mathrm{x}<\mathrm{D} \\
1, & \mathrm{x}>\mathrm{D}\end{cases} \\
& \mathrm{x} \equiv \frac{\mathrm{r}}{\mathrm{r}_{\mathrm{m}}} ; \mathrm{r}_{\mathrm{m}}=4.008 \AA ; \quad \mathrm{A}^{*}=1.10146811 ; \quad \alpha^{*}=9.39490495 ; \quad \beta^{*}=-2.32607647 ; \mathrm{D}=1.28 ; \quad \mathrm{C}_{6}=1.08822526 ; \mathrm{C}_{8}= \\
& 0.53911567 ; \mathrm{C}_{10}=0.42174119 ; \varepsilon / \mathrm{k}_{\mathrm{B}}=201.2 \mathrm{~K} \text {. }
\end{aligned}
$$

\section{Results and Discussion}

Our results are summarized in Figures 1-2. The principal physical quantities here are the total and viscosity effective cross sections (i.e., in the medium), which are calculated using Equations (1) and (2), respectively. These are calculated using the HFD-B potential. Figure 1 represents the effective total cross section $\sigma_{\mathrm{T}}$ for $\mathrm{Kr}-\mathrm{Kr}$ scattering at a boiling point as a function of $\mathrm{k}$ in the medium [GMF]

As seen in the figure, the cross section have a peak at a particular energy. The peaks were refered to as resonances. The resonances are essentially bound states, but with shorter lifetimes. In other words, they are quasi-bound states. These arise because the repulsive angular-momentum barrier $\sim \frac{\ell(\ell+1)}{\mathrm{r}^{2}}$ 'screens' the short-range repulsive part of the interatomic potential, thereby allowing the interacting particles to 'see' in effect more attraction. The minimum is evidence for the Ramsauer-Townsend effect (Feltgen et al., 1973), which is a physical phenomenon occurring in the collision between two particles when the total cross section is a minimum and, therefore, the mobility is a maximum. In the high-energy region, there are undulations in $\sigma_{\mathrm{T}}$. These originate from the indistinguishability of $\mathrm{Kr}$ atoms, which are scattered by the repulsive part of the potential. In the high-energy region, there are undulations in $\sigma_{\mathrm{T}}$. These originate from the indistinguishability of $\mathrm{Kr}$ atoms, which are scattered mainly by the repulsive part of the potential. Since the kinetic-energy part is much larger than the interaction part, the amplitude of the undulations decreases, to a first approximation, as the inverse of the relative velocity of the colliding atoms.

At $\mathrm{k}<2.6 \AA^{-1}$, corresponding to relatively large interatomic spacing $\mathrm{r}$ : the $\mathrm{Kr}-\mathrm{Kr}$ interaction becomes attractive. At $\mathrm{k}>2.6 \AA^{-1}$, corresponding to relatively small $\mathrm{r}$, the $\mathrm{Kr}-\mathrm{Kr}$ interaction becomes repulsive.

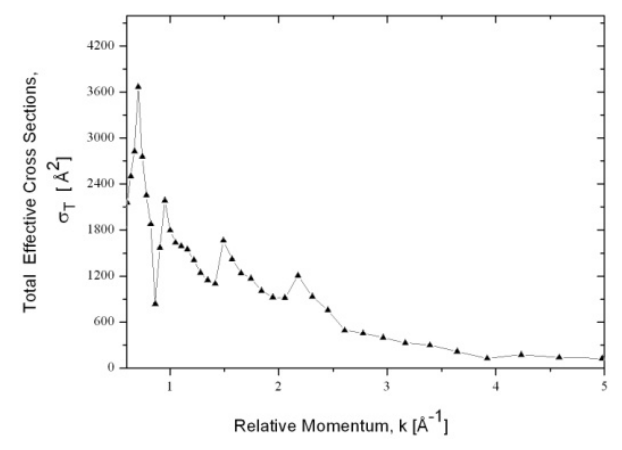

Figure 1. The effective cross section $\sigma_{\mathrm{T}}$ for $\mathrm{Kr}-\mathrm{Kr}$ scattering as a function of relative momentum $\mathrm{k}\left[\AA^{-1}\right]$ Figure 2 represents the effective viscosity cross section $\sigma_{\eta}$ as a function of $k$; $\sigma_{\eta}$ has the same overall behavior as $\sigma_{\mathrm{T}}$. 


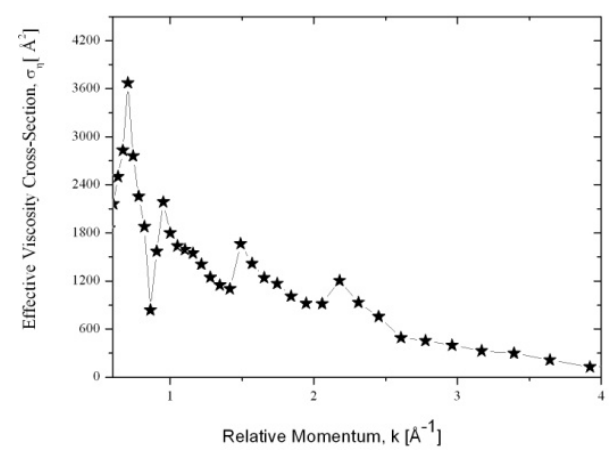

Figure 2. The effective viscosity cross section $\sigma_{\eta}$ for $\mathrm{Kr}-\mathrm{Kr}$ scattering as a function of relative momentum $\mathrm{k}\left[\AA^{-1}\right]$

\section{Conclusion}

The achievements of this work are: (1) the calculation of the total and viscosity cross sections for krypton gas at boiling point by using a Galitskii-Migdal-Feynman (GMF) formalism which is essentially an independent-pair model 'dressed' by a many-body medium. The interaction potential in our work is theHartree-Fock dispersion (HFD-B) potential, (2) the prediction of resonance-like behavior and the Ramsauer-Townsend effect in $\mathrm{Kr}$ gas.

\section{Conflict of interests}

The authors declare that there is no conflict of interests regarding the publication of this paper.

\section{References}

Al-Barghouthi, H. M. (1997). A Microscopic Study for Neutral Many-Bosinc Systems. Unpublished Master Thesis, University of Jordan, Amman, Jordan.

Bishop, R. F., Ghassib, H. B., \& Strayer, M. R. (1976), Composite Pairs and Effective Two-Body Scattering in a Many-Body Medium. Physical Review A, 13, 1570-1580.

Feltgen, R., Pauly, H., Torello, F., \& Vehmeyer, H. (1973), Determination of the 4He-4He repulsive Potential up to $0.14 \mathrm{eV}$ inversion of high-resolution total-cross-section measurements. Physical Review Letters, 30 , $820-823$.

Fetter, A. L., \& Walecka, J. D. (1971). Quantum Theory of Many-Particle Systems. New York: McGraw-Hill.

Geltman, S. (1997). Topics in Atomic Collision Theory. Florida: Krieger Publishing Company.

Ghassib, H. B., Bishop, R. F., \& Strayer, M. R. (1976). A Study of the Galitskii-Feynman T-Matrix for Liquid 3He. Journal of Low Temperature Physics, 23, 393-401.

Joudeh, B. R. (2013). Scattering Properties of Ground-State Spin-Polarized Atomic Hydrogen. Physica B: Condensed Matter, 421, 41-45.

Kanzleiter, R. J., Stotler, D. P., Karney, C. F. F., \& Steiner, D. (2000). Improved elastic Collision Modeling in DEGAS 2 for Low-Temperature Plasmas. Phys. Plasmas, 7, 5064-5069.

Kittel, C., \& Kroemer, H. (1980). Thermal Physics. New York.

Merzbacher, E. (1998). Quantum Mechanics (3rd ed.). New York: Wiley.

Robert, P. M., \& Stauffer, A. D.(2015). Viscosity cross sections for the heavy noble gases. The European Physical Journal D.

Ronald, A. A., \& Slaman, M. J. (1986). The argon and krypton interatomic potentials revisited. Molecular Physics, 58(4), 679-697.

Wright, M. J., Bose, D., Palmer, G. E., \& Levin, E. (2005). Recommended Collision Integrals for Transport Property Computations. Part 1: Air Species AIAA Journal, 43, 2558-2564.

\section{Copyrights}

Copyright for this article is retained by the author(s), with first publication rights granted to the journal.

This is an open-access article distributed under the terms and conditions of the Creative Commons Attribution license (http://creativecommons.org/licenses/by/4.0/). 